\title{
SIMULATION-BASED ASSESSMENT OF ELECTRIC LOAD MANAGEMENT PROGRAMS
}

\author{
A. GOMES ${ }^{1}$, A. GOMES MARTINS ${ }^{1 *}$ AND R. FIGUEIREDO ${ }^{2}$ \\ ${ }^{1}$ Department of Electrical Engineering, FCTUC/INESC, Polo 2 University of Coimbra, Pinhal de Marrocos, 3030 Coimbra Portugal \\ ${ }^{2}$ Department of Mechanical Engineering, FCTUC, Polo 2 University of Coimbra, Pinhal de Marrocos, 3030 Coimbra Portugal
}

\begin{abstract}
SUMMARY
The paper describes a methodology for assessing the contribution of storage type loads to an electric energy system's load curve and for evaluating the impact of load shedding actions, as part of load management programs, on the system's load curve. The methodology uses physically based individual load models and an aggregation procedure that accounts for both statistical reference patterns of the energy service demand and for the random nature of load use. A simulation case study of electric water heaters fed by a single transformer station is presented, to illustrate the effects of load control. The proposed methodology presents the possibility of evaluating — both at individual consumer level and at system levelthe impacts of load management (LM) on the quality of service provided, besides anticipating the impact on the system's load curve. It allows an effective design of LM programs through variables such as the number of different load shedding schedules and their frequency of use by the utility. Copyright (C) 1999 John Wiley \& Sons, Ltd.
\end{abstract}

KEY WORDS demand-side management; load models; load management; simulation; load aggregation; payback effect

\section{INTRODUCTION}

Any demand-side management (DSM) program needs previous load research to appraise the main characteristics of loads and estimate the potential effects of DSM measures. Load research is a costly activity as it often requires pilot actions to be carried out, with data being collected in various ways before, during and frequently after the implementation of each action. Furthermore, when pilot actions involve remote control loads - or load management ( $\mathrm{LM}$ - - it is also necessary to evaluate dynamic load response to shedding and restoration of supply.

Previous appraisal of the various possible kinds of effects is crucial for a correct evaluation of LM programs in the context of a portfolio of DSM options, particularly when a utility uses integrated resource planning (IRP) as its planning methodology. The duration of load shedding actions, as they are applied mainly to storage type loads - e.g. electric water heaters or HVAC - , depends strongly on the energy storage capacity of these loads. On the other hand, the final effect on the utility's load curve (LC) may differ from the goal set at the beginning if appropriate assessment of load's behaviour in response to LM is not made in a detailed manner. This assessment must also take into account the eventual quality of service decrease caused by LM to final users as a result of too long unavailability of loads.

Hence, having a means of anticipating LM effects is a goal sought by power system planners and technical staff involved in network management and control, from two perspectives - the system's LC modifications

\footnotetext{
* Correspondence to: A. G. Martins, Department of Electrical Engineering, FCTUC/INESC Polo 2 University of Coimbra, Pinhal de Marrocos, 3030 Coimbra Portugal. Email: amartins@dee.uc.pt

Contract grant sponsors: Junta Nacional de Investigação Científica e Tecnológica and the Portuguese Ministério da Ciência e Tecnológica
}

CCC 0363-907X/99/020169-13\$17.50

Received 22 June 1998

Copyright (C) 1999 John Wiley \& Sons, Ltd.

Accepted 24 July 1998 
and the final user's expectations (both economical and concerning quality of supply). Simulation is gradually proving to have the potential to satisfy this goal. Not only it allows e.g. the detection and measuring of the so-called 'pay-back' effect, but also the measuring of effects on variables that may be used to assess impact on consumer's comfort.

Load modelling is discussed in the paper, along with the use of load models in simulation studies that may help designing LM programs. An adequate LM program is one that serves the utility's goals by anticipating effects of shedding and restoration of supply to loads, having in mind customer-side constraints. A simulation tool must allow rehearsing different control strategies in order to identify the ones considered to be the most appropriate.

\section{LOAD MODELLING}

Some load models are based on a representation of the physical phenomena associated to load's operation, as in Chong and Malhamé (1984) and Alvarez et al. (1992). The utilization of energy services by consumers has a random nature - the events of switching loads on and off has no predetermined time schedule. Also, other factors with strong influence on energy consumption patterns have a clear random nature - as for example the weather conditions, which influence the use of HVAC services. Thus, the use of physically based load models (PBLM) to obtain feasible representations of certain classes of demand behaviour at system level is not a straightforward task. It is possible to find several individual load models reported in the literature, as in Myers et al. (1983), Lee and Wilkins (1983), Chong and Malhamé (1984), Chong and Debs (1979), Gustafson and others (1992) and Pahma and Brice (1985) - based on the analytical treatment of thermal energy exchange between two different systems. As it is also possible to find several load models, at system level, with various degrees of aggregation (the consideration of groups of individual loads) (Gellings and Taylor, 1981) (Rau and Graham, 1979) (Laurent and Malhamé, 1993) (Capasso et al., 1993). Aggregation may be achieved in a bottom-up approach, based on individual load models, or through the consideration of small groups of similar loads with similar consumption patterns, which are more easily simulated at system level. This approach is suggested by Chong and Malhamé (1984) and by Chong and Debs (1979). In general, the use of PBLM provides a means of assessing not only the contribution of the modelled loads to the system's load curve (LC), but also of assessing the effects of eventual remote load control actions on the LC. Usual load modelling approaches, mostly for load forecast, system planning or load flow studies, are not adequate for these purposes because they tend to represent the system and its loads in a 'normal' or 'uncontrolled' state, meaning that no effects of load control actions are accounted for. Regression analysis, which is the basis of many such load models, is not adequate outside the scope for which it is traditionally used. Only PBLM allows, e.g. capturing the payback effect associated with the restoration of supply to a group of thermostatically controlled loads after a period of interruption of supply, which may lead to an important localized power demand.

\section{PHYSICALLY BASED INDIVIDUAL LOAD MODELS}

The load models discussed in the paper are based on the energy balance which exists in water heating (WH) systems. Thermal energy exchange is due to the need of maintaining the water in the tank at a certain temperature and also to the need of heating the incoming water when there is extraction from the tank for any use. The WH demand is determined by the temperature of the water inside the tank, in such a way that the heating element is powered when the temperature falls below the lower limit of the thermostat deadband and it is disconnected when the temperature reaches the higher limit of the thermostat deadband. The WH model tracks the water temperature value to define the load profile. This model has been described in Gomes and Martins (1995). Figure 1 represents one graphical output of the individual WH load model. The abscissa axis represents time, from 0 to $24 \mathrm{~h}$. The other axis represents either power in $\%$ of rated value or temperature 


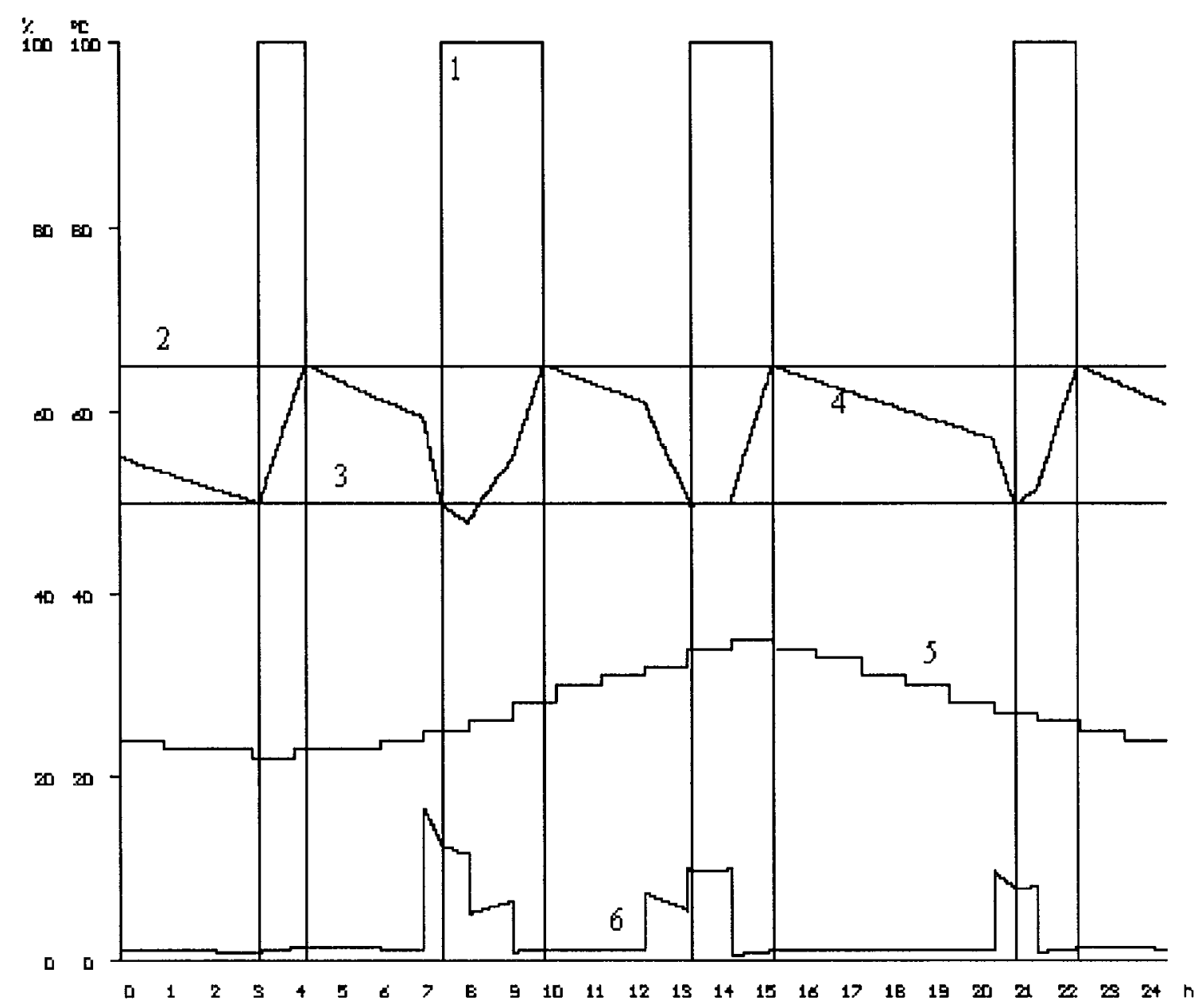

Figure 1. A graphical output of the individual WH load model

in degrees ${ }^{\circ} \mathrm{C}$. The curves have the following meanings:

1. WH load pattern,

2. higher temperature limit of the dead-band,

3. low limit of the dead-band,

4. evolution of the water temperature inside the WH tank,

5. temperature of the air surrounding $\mathrm{WH}$,

6. evolution of the energy which is extracted per unit time due to losses, and to hot water demand - the curve is scaled to $1 / 10$.

The model referred to provides information which is enough to assess the possible adverse effects of load control at end-user level. In fact, as water temperature inside the tank is 'monitored', it is possible to define thresholds of comfort - or discomfort - as an image of final user's tolerance to load control effects.

\section{AGGREGATION OF INDIVIDUAL MODELS - A METHOD}

The random nature of the factors that influence load use is one of the greatest difficulties to overcome when simulating load behaviour at system level. Nevertheless, aggregation may be considered as having a filtering 
feature that reduces the importance of this randomness, apparently in a way that depends directly on the number of loads - the higher the number, the more important the filtering effect. The main purpose of using individual PBLM, besides the ease with which LM actions can be implemented is to obtain the aggregated LC. Each parameter potentially subject to random variations is assumed to have a 'typical' (or average) value or pattern which is used as the basis to determine the values assigned to each individual load, according to a predetermined distribution (usually a normal distribution). This 'typical' or reference value or pattern is obtained either through data gathering in load research campaigns or in statistical files, according to the nature of the parameter. Differing from other modelling approaches, in the one described here parameters are allowed to vary on an hourly basis. Instead of assuming a constant value for as long a period as, e.g. a whole day, or of assuming values according to some probability distribution type, some parameters are made to follow a reference pattern with a possibly different seed value for each hour, defined according to the outcome of a probability distribution, valid for that hour only.

The use of hourly patterns is not an obstacle to the use of the models proposed when data from load research campaigns are available. On the other hand, variables whose values are not necessarily obtained in such campaigns, as e.g. environmentally related ones, are usually available on an hourly basis. Besides, the use of such a time basis facilitates the extension of these models to a smaller number of loads than is traditionally possible with other aggregation methods that rely on a great number of load points for feasibility. As these models are physically based and average values are based on collected data, a moderate amount of simulation runs is needed to obtain the aggregated LC when a small number of load points is addressed. Figure 2 depicts a daily average hot water consumption pattern obtained for a sample of residential consumers at the city of Coimbra. As mentioned above, the hourly values have been obtained by using an average reference pattern as a framework and a seed value for each hour obtained, in this case, as the outcome of a normal probability distribution. In the beginning of the simulation process, if the initial temperature of the water inside each EWH is within the lower limits of the deadband, both the temperature of the hot water and the on/off EWH state are assigned a random value at the beginning of the simulation interval.

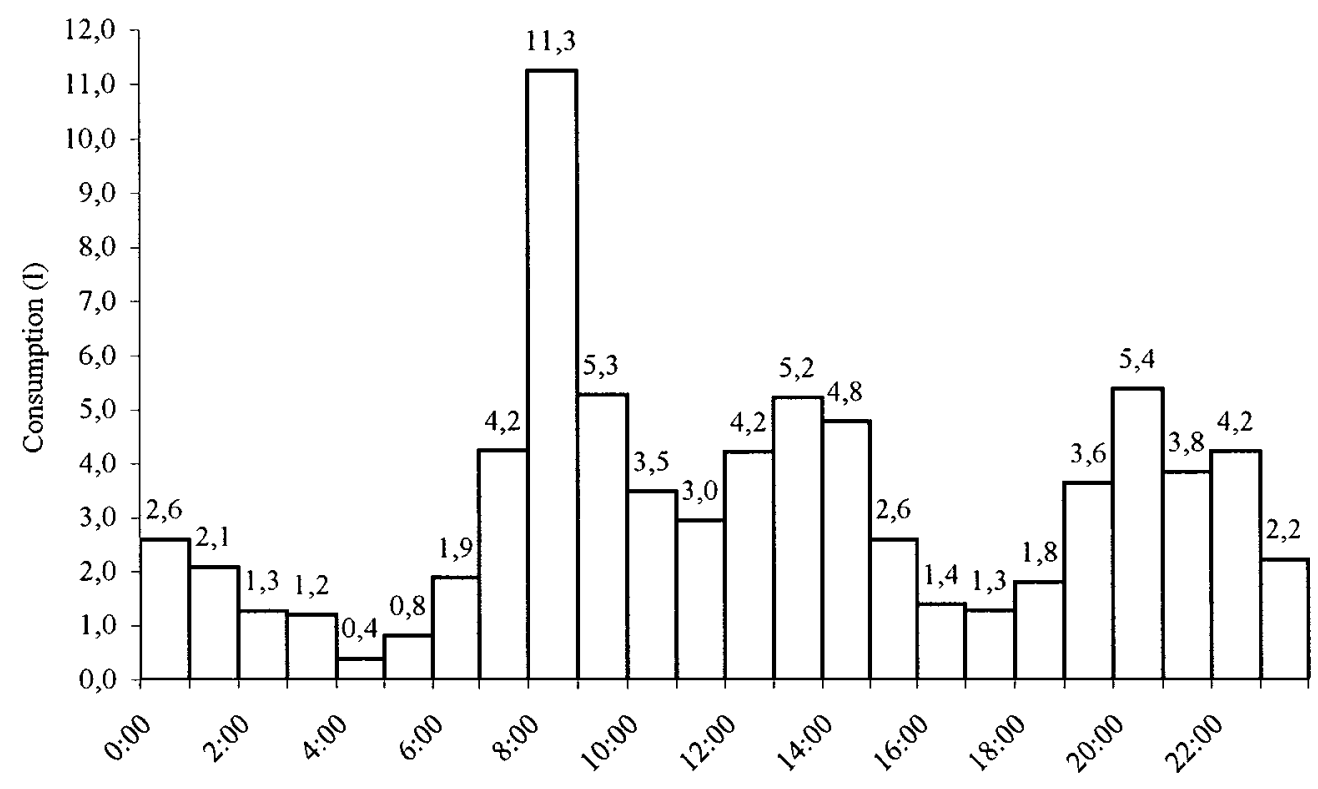

Figure 2. Daily average values of hot water consumption obtained through LD of EWH 


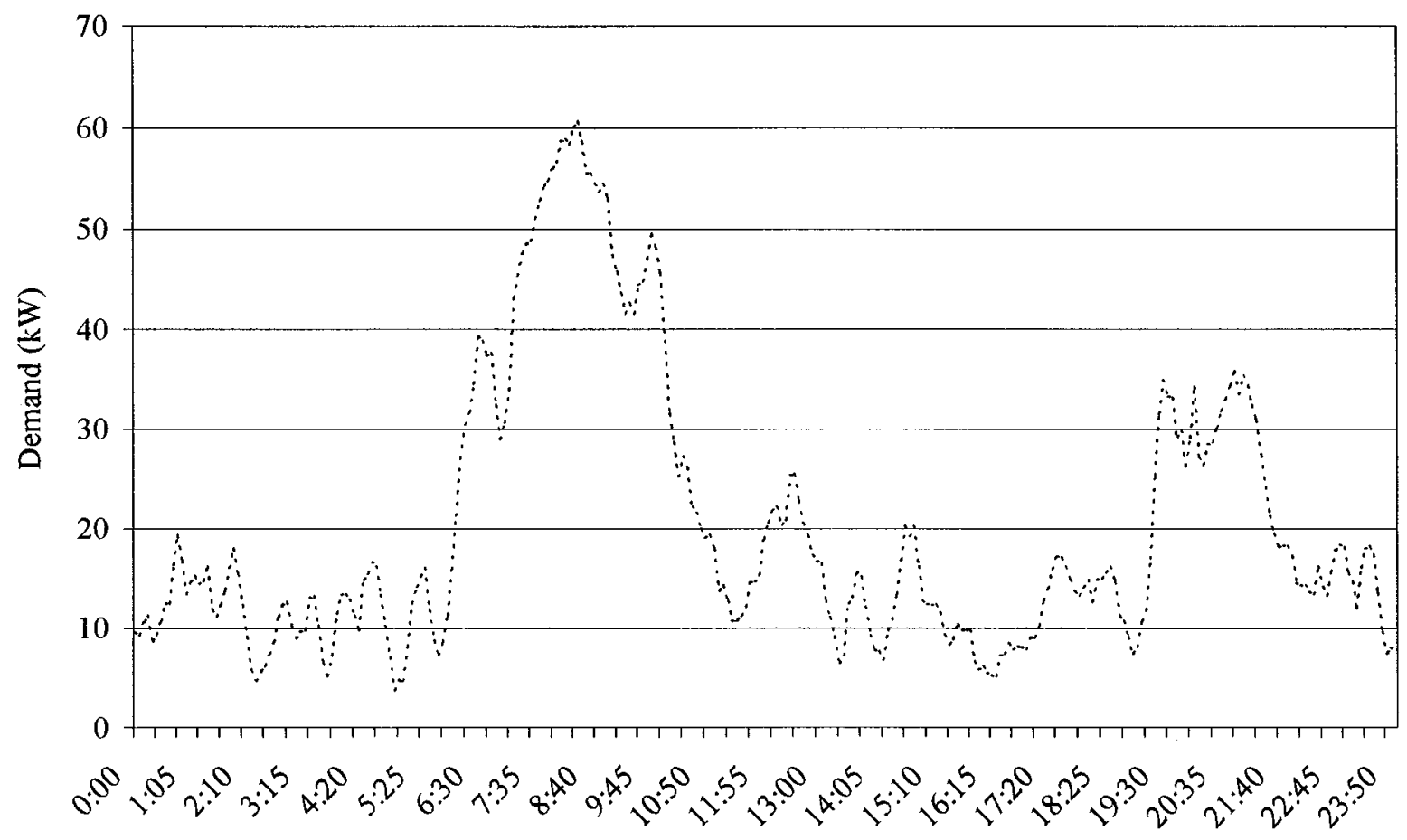

Figure 3. Demand curve of the simulated EWH

A simplified sequence of the operations involved in building an aggregated model of, e.g. electric water heaters $(\mathrm{EWH})$ may be as follows:

- determination of hot water usage patterns,

- identification of end-user groups according to the type of use of hot water.

- definition of a sample as a representation of the total universe of loads - the sample magnitude defines the number of simulations of individual loads to be carried out

- simulation of the loads in the sample, using a normal distribution with a mean of 1 and std. dev. of 1 for hot water demand variations from each EWH to the next EWH. These unitary values correspond to a compromise that proves to be adequate (cf. Figure 3) in view of the LC obtained through data collection.

One of the outcomes of the simulation process is the demand curve of the modelled type of load, as in Figure 3. In this case a group of $50 \mathrm{EWH}$ has been simulated. With the tool developed to support the described method it is possible to simulate different patterns of water consumption and family sizes.

The method proposed is complemented with the simulation of shedding actions imposed on the loads, adequately organized in groups of individual loads that are shed and restored together. This simulation is carried out under similar conditions to the first one, so that it is possible to compare system's load curve in both situations - without and with load control. The impacts of load control actions can be easily foreseen because they can be incorporated in individual PBLM.

\section{A SIMULATION BASED CASE-STUDY}

A small urban area has been chosen to exemplify the application of the above-described methodology. It is located at the historical centre of the city of Coimbra, in Portugal. It is fed by a transformer station (TS) 
serving a peak load which is near its installed capacity. This TS feeds 177 customers, 121 of them being residential customers representing about $42 \%$ of total consumption, 29 commercial customers with a share of about $10 \%$ of consumption and 27 customers of different types. About $41 \%$ of the residential consumers possess electric water heaters, according to recent statistical data available. The composition and relative importance of the 50 families in these circumstances are depicted in Table 1.

The TS global load curve is shown in Figure 4. Batch simulations of EWH have been performed, according to the methodology described, in order to assess the contribution of these loads to the global LC and using the average hot water consumption presented in Figure 2. The resulting daily demand curve (DDC) is depicted in Figure 5, along with the global LC. Maximum demand occurs in the morning and is mainly due to residential consumption. As these high demand values last for a number of hours, several groups of loads are needed to control demand without high discomfort levels to consumers and to avoid the so-called 'payback effect'.

Four different homogeneous groups of loads have been identified and several shedding actions of equal duration, but spread throughout time, have been applied to these groups. The factors normally used to define

Table 1. Number and composition of the families with EWH

\begin{tabular}{lcccc}
\hline Type & Size of each family & \% of families & No of families & No of persons \\
\hline T1 & 1 & 20 & 14 & 14 \\
T2 & 2 & 41 & 29 & 58 \\
T3 & 3 & 31 & 22 & 66 \\
T4 & 4 & 5 & 4 & 16 \\
T5 & 5 & 3 & 2 & 10 \\
\hline
\end{tabular}

Load Curve of the Transformer Station

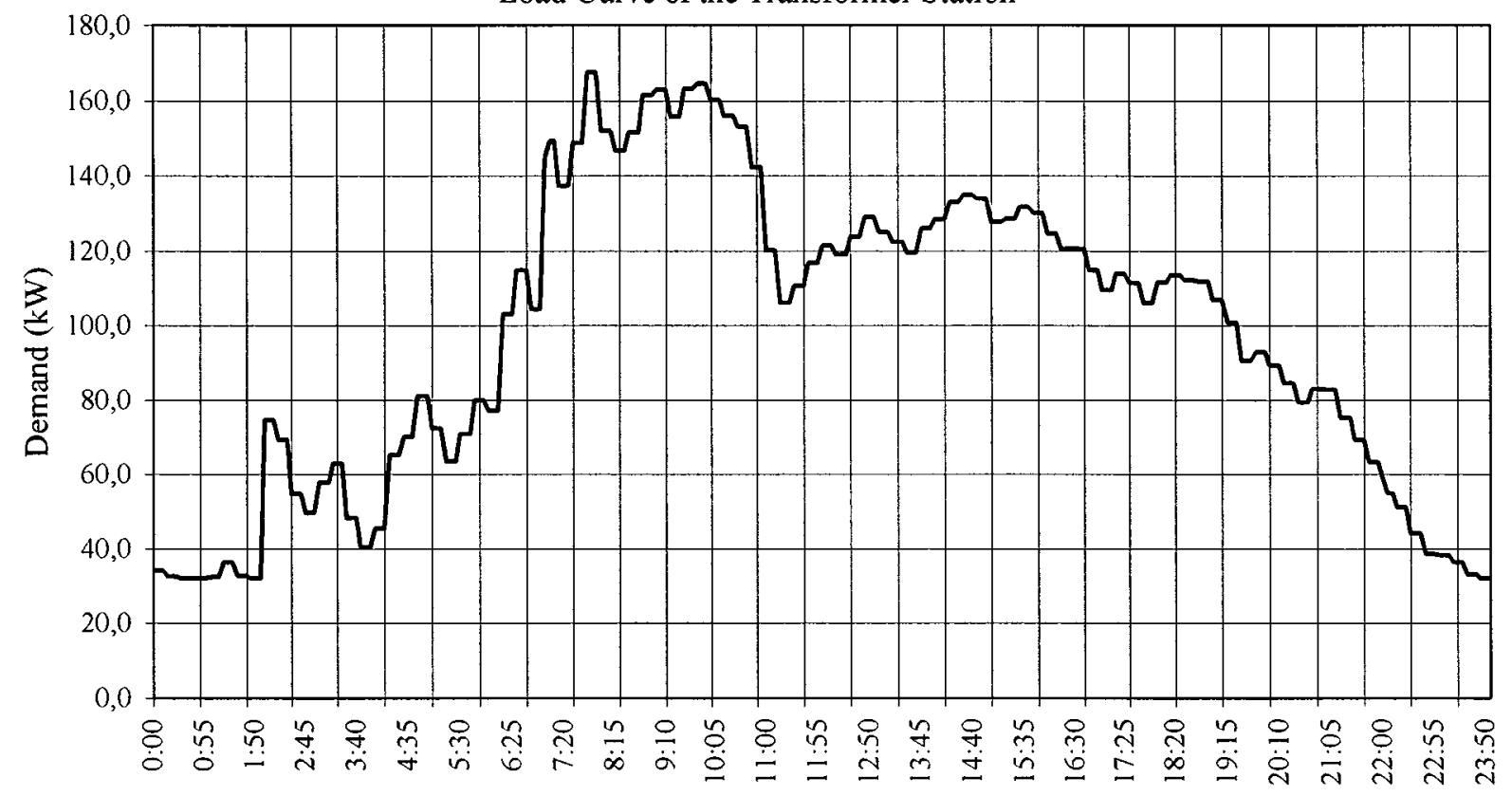

Figure 4. LC of transformer station 


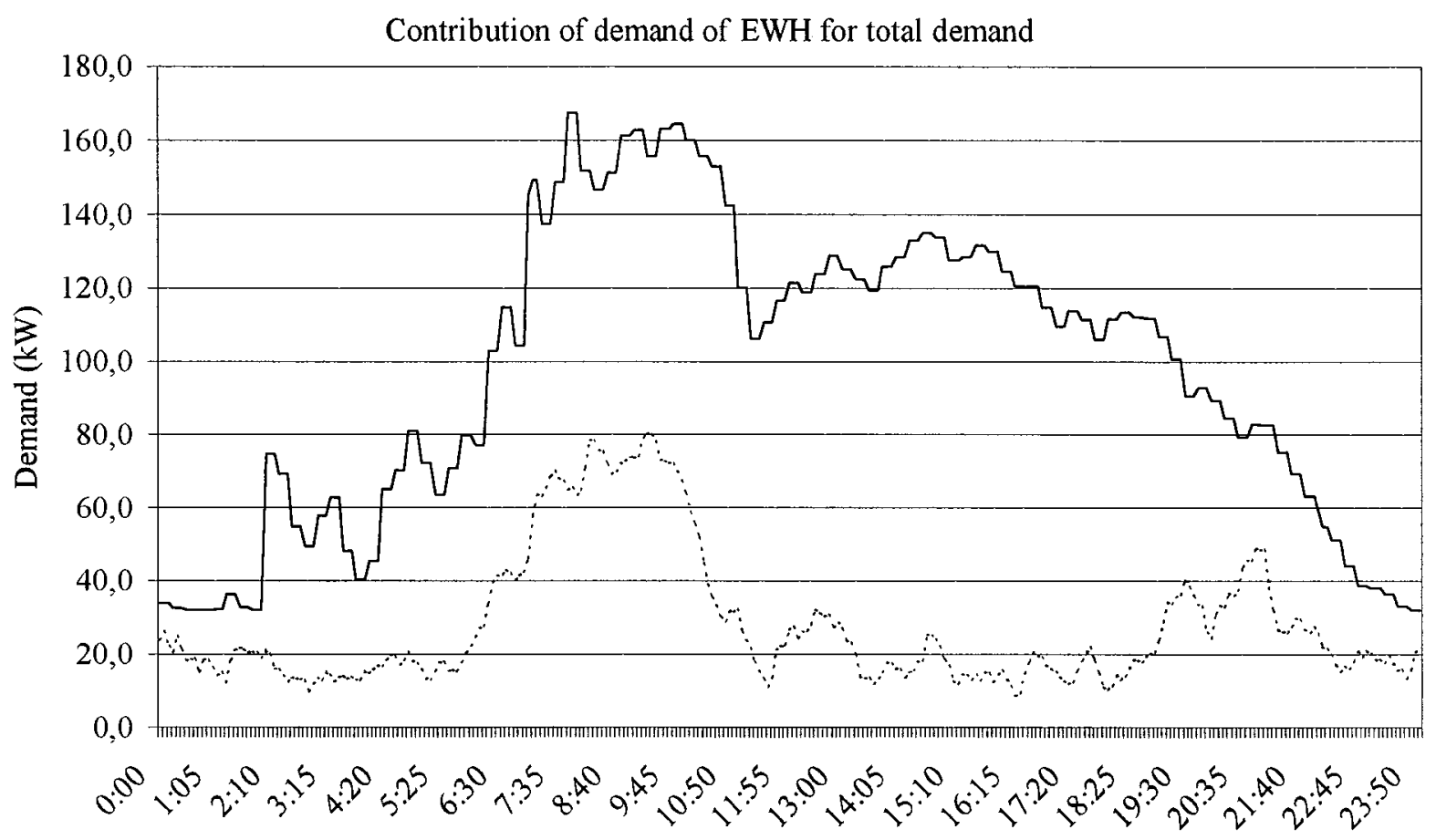

Figure 5. Contribution of LC of EWH for LC global

Table 2. Composition of EWH groups

\begin{tabular}{lrrrrl}
\hline Type of family & T1 & T2 & T3 & T4 & T5 \\
\hline Group 1 & 3 & 5 & 4 & 0 & 1 \\
Group 2 & 2 & 5 & 4 & 1 & 1 \\
Group 3 & 3 & 5 & 3 & 1 & 0 \\
Group 4 & 2 & 5 & 4 & 1 & 0 \\
Total & 10 & 20 & 15 & 3 & 2 \\
\hline
\end{tabular}

homogeneous groups of loads are: size of individual families, equipment characteristics, environmental conditions and geographical location. The latter two do not have a great influence in this example because the loads under consideration are located in the same urban area and, therefore, subject to the same environmental conditions. Besides, a single EWH model has been used in the simulations, as it corresponds to the most abundant in this area-801/1.5 $\mathrm{kW}$.

The main objective of this case study is solely to present the methodology without any special concern about the composition of the control groups, or about the adequacy of the scheduling of the shedding actions. Moreover, the consideration of control groups in itself, as opposed to a single set of simultaneously switched EWH, is due to the need of avoiding high demand values from the payback effect. The possibility of gradually restoring supply to previously shed devices allows a smoother evolution of demand. The groups have been defined by means of an even distribution of the 50 families whose EWH have been considered as remotely controllable as shown in Table 2 .

Table 3 depicts the schedules used for load shedding, where each period of supply interruption to a group of loads is a multiple of $15 \mathrm{~min}$, never exceeding $30 \mathrm{~min}$. 
Table 3. Scheduling of control actions

\begin{tabular}{|c|c|c|c|c|c|c|c|c|c|c|c|c|c|c|c|c|}
\hline Type of family & $\frac{n}{\ddot{r}}$ & 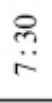 & $\stackrel{\stackrel{n}{\vartheta}}{\check{r}}$ & $\underset{\infty}{\stackrel{8}{\infty}}$ & $\frac{n}{\infty}$ & $\begin{array}{l}\stackrel{\oplus}{\dddot{\infty}} \\
\text {. }\end{array}$ & $\begin{array}{l}\stackrel{?}{q} \\
\ddot{\infty}\end{array}$ & $\begin{array}{l}8 \\
\ddot{\sigma}\end{array}$ & $\frac{n}{a}$ & $\begin{array}{l}\stackrel{\rho}{~} \\
\ddot{\sigma}\end{array}$ & $\begin{array}{l}\stackrel{n}{*} \\
\ddot{\alpha}\end{array}$ & $\begin{array}{l}8 \\
\ddot{0}\end{array}$ & $\frac{n}{\ddot{\theta}}$ & $\begin{array}{l}\stackrel{्}{\ominus} \\
\ddot{O}\end{array}$ & $\begin{array}{l}\stackrel{9}{+} \\
\stackrel{0}{0}\end{array}$ & $\begin{array}{l}\stackrel{8}{\ddot{ }} \\
=\end{array}$ \\
\hline Group 1 & & & & & & & & & & & & & & & & \\
\hline Group 2 & & & & & & & & & & & & & & & & \\
\hline Group 3 & & & & & & & & & & & & & & & & \\
\hline Group 4 & & & & & & & & & & & & & & & & \\
\hline
\end{tabular}

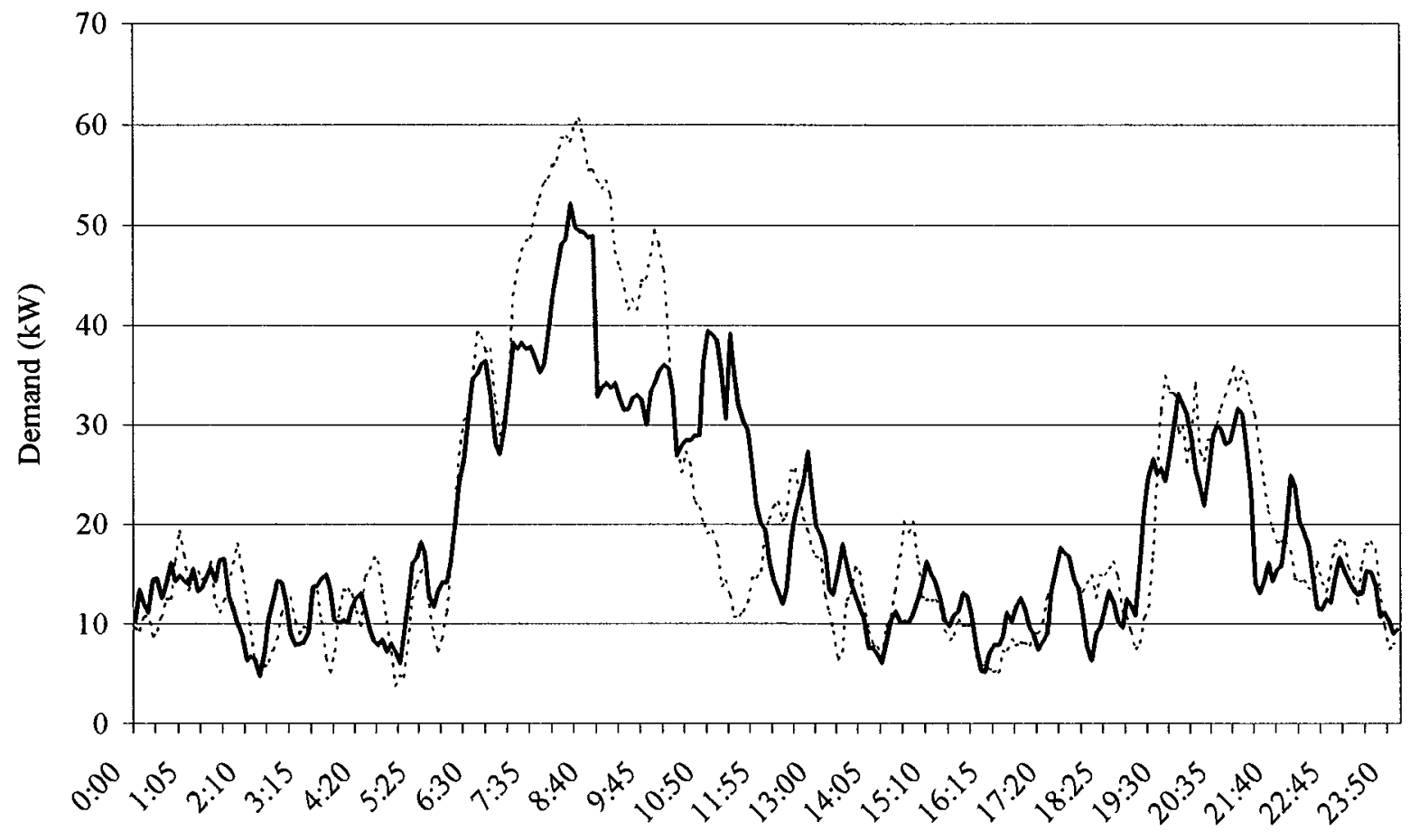

Figure 6. Load curve of EWH with and without control

In general, several iterations must be performed, with as many simulations as needed in order to achieve a satisfactory peak demand reduction without violating end-user related constraints -namely comfort, or the quality level of the energy service provided. With the information available after the simulations it is possible to evaluate the effects both at end-user and utility level.

\section{CASE STUDY RESULTS}

Two batch simulations have been carried out. As a result the load curves of EWH with and without load control have been obtained, both on a basis $5 \mathrm{~min}$ average power. The 'load shifting' effect is significant and quite evident in Figures 6, 8 and 9.

Figure 7 depicts the differences between the LC of EWH with a peak clipping strategy and the LC of EWH without any control action. A shifting of consumption from the period 7:00-10:00 h to the period 


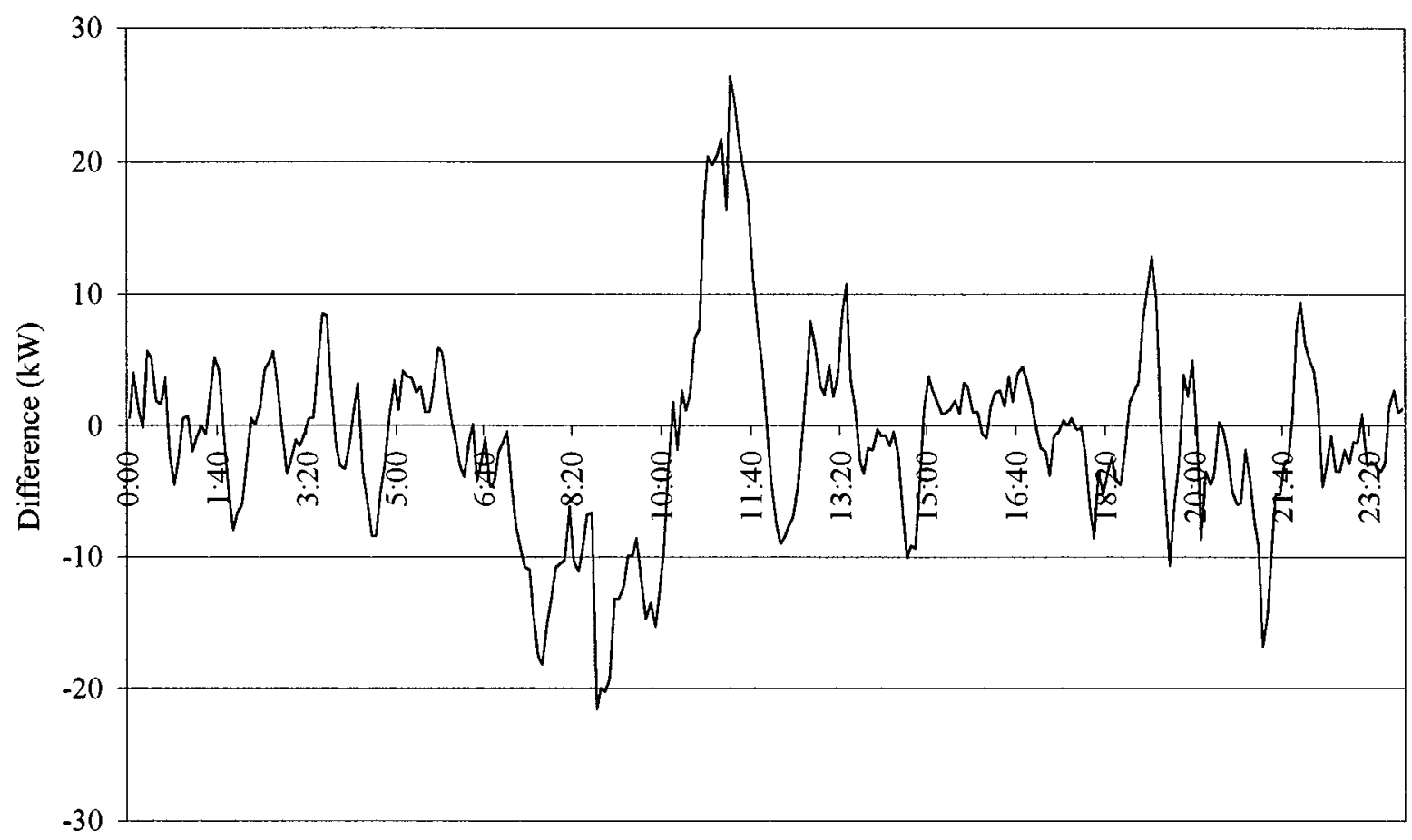

Figure 7. Difference between the LC of EWH with and without control

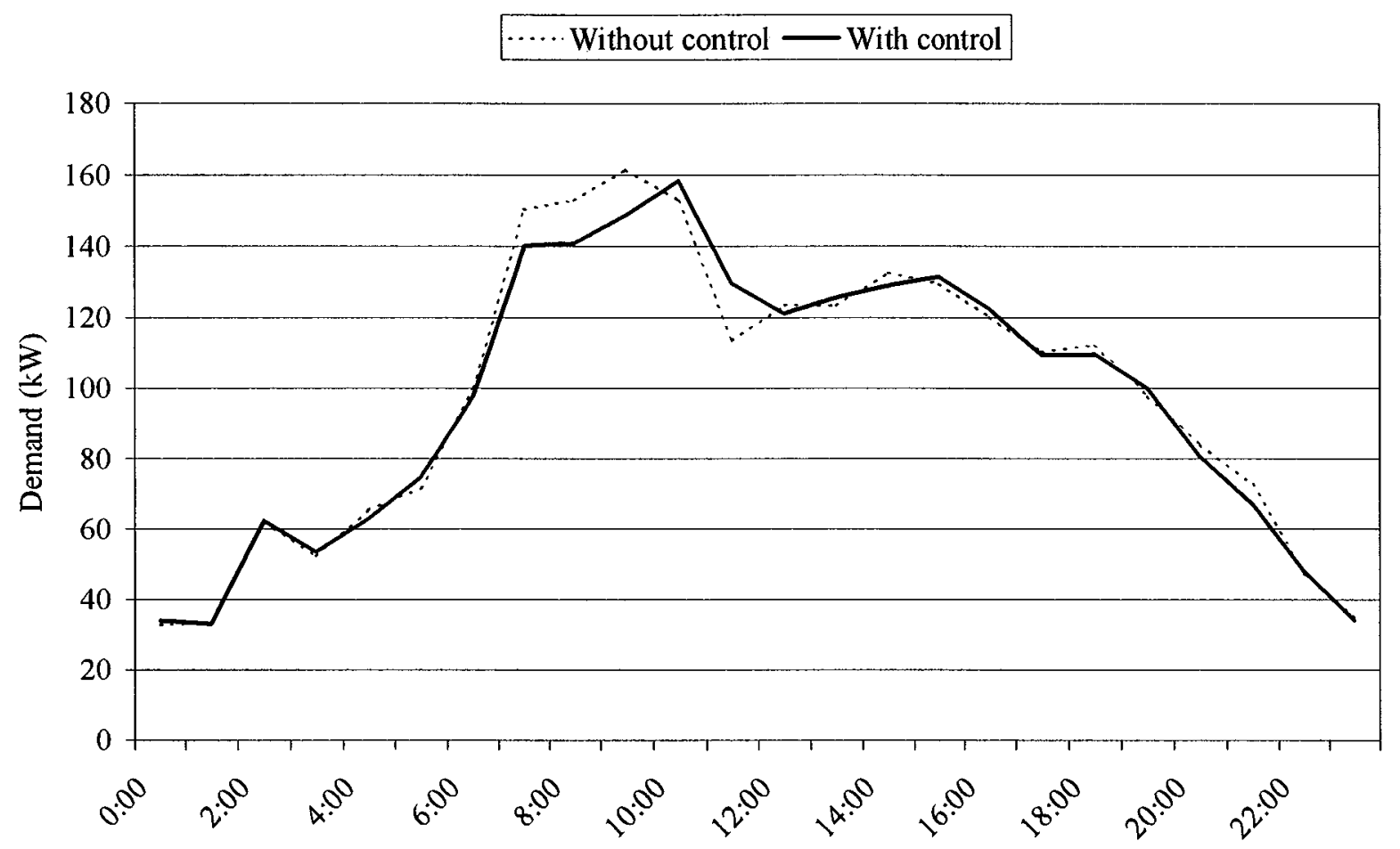

Figure 8. Diversified load curve with and without control, on an hourly basis 


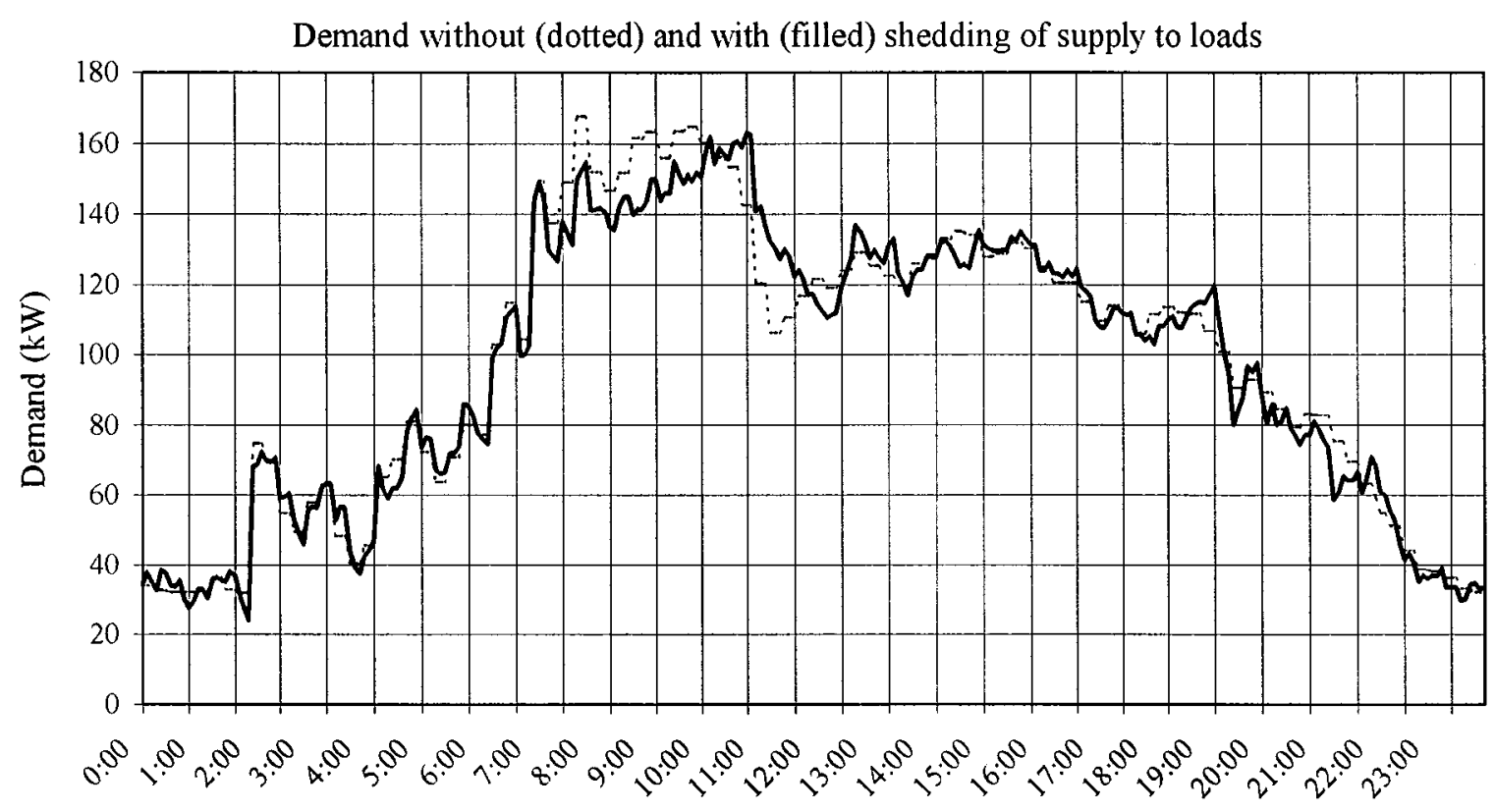

Figure 9. Demand before and after the shedding of supply to loads

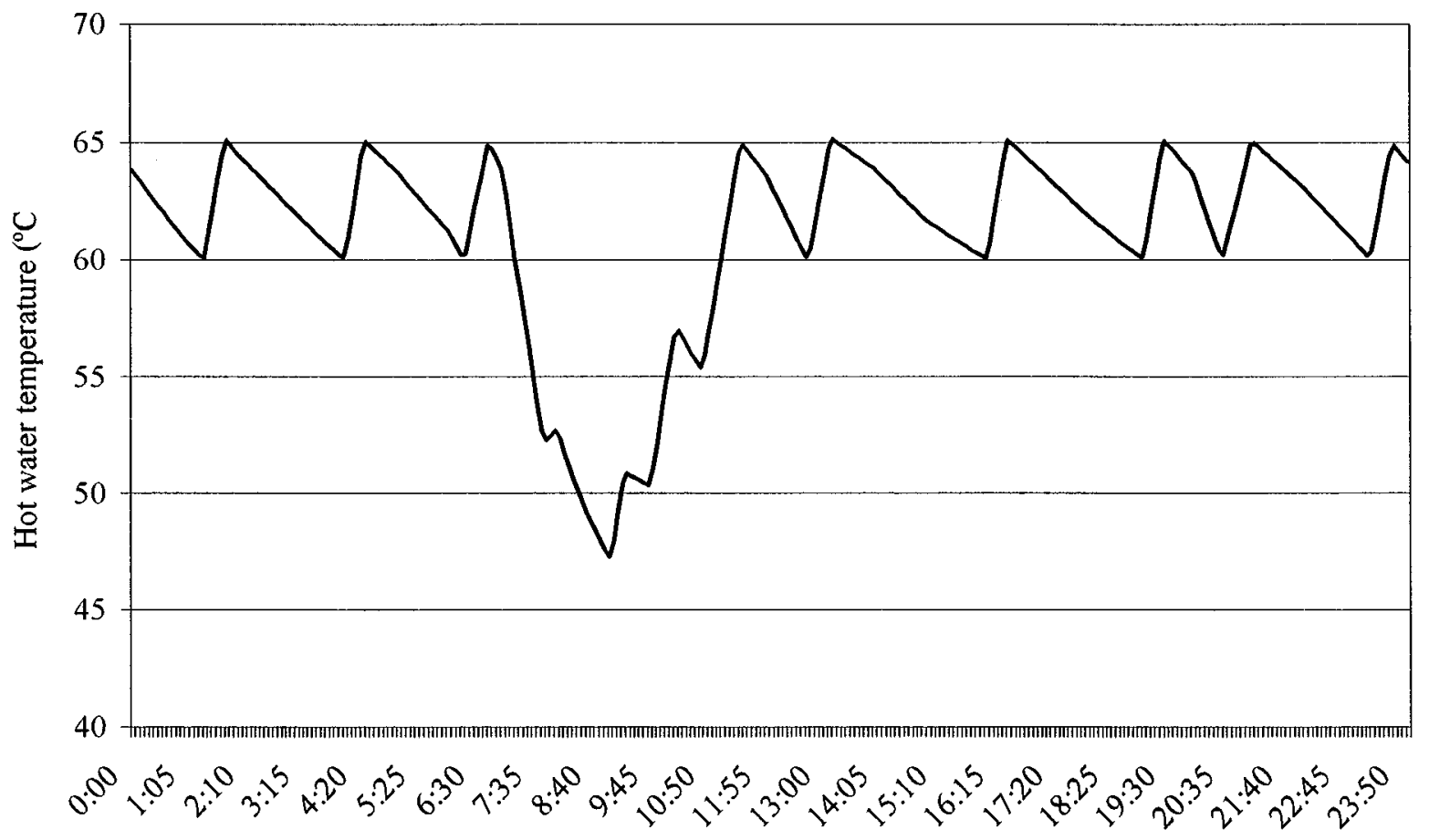

Figure 10. Hot water temperature in the EWH with the lowest minimum value 


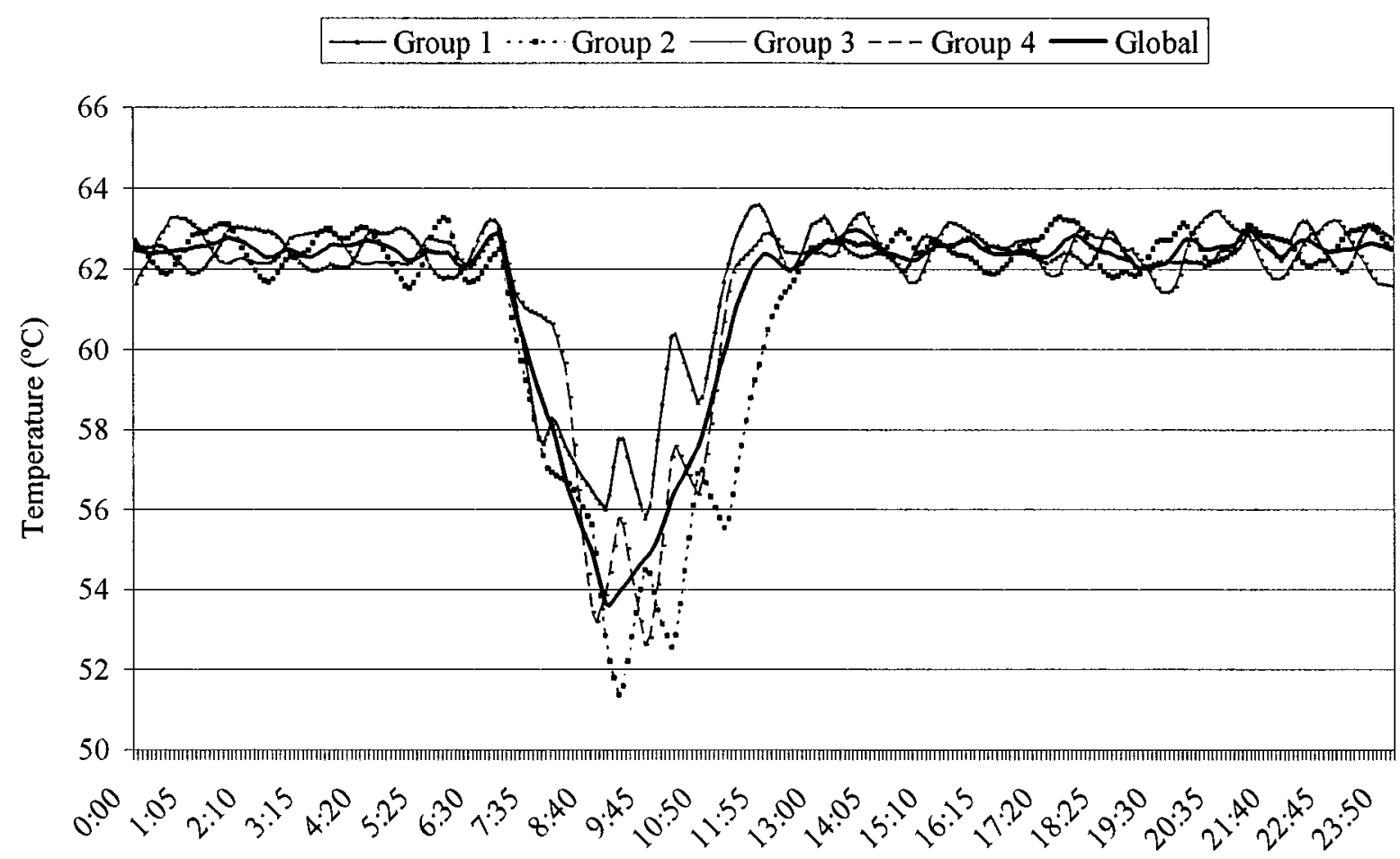

Figure 11. Average water temperatures of the groups of EWH

10:00-12:00 h can be observed. Restoration of supply to loads produces a peak that must not be neglected, and both the composition of the control groups and the definition and time schedule of the control actions must be carefully designed.

The demand of EWH with and without shedding of supply to loads is presented in Figures 8 and 9. The effect on the TS load curve is quite visible, in Figure 9, with a maximum demand reduction of $5 \mathrm{~kW}-3,5 \%$ of the uncontrolled value - which partially relieves the distribution transformer burden in spite of the restoration peak (Figure 7).

The results could have been different (and probably improved in terms of maximum demand reduction) if the composition of the goups and the time schedule of the control actions had been devised with some kind of optimization strategy.

Finally, the quality of water heating service provided to the customers can be assessed, as the simulation tool retains the results pertaining to each individual EWH. Among these, water temperature is an adequate measure of the quality of the energy service. Figure 10 represents the evolution of the water temperature inside the tank of the worst case EWH. On the other hand, a global assessment of the impact of load control on consumers' comfort may be drawn from the average temperatures of the four control groups and the global average temperature, that are represented in Figure 11.

\section{CONCLUSION}

The approach to load aggregation described in the paper has the advantage of being based on physically based models with hourly discretization of the level of energy service used (in the present case, corresponding 
to hot water consumption). This particular time resolution (hourly) is common to a great number of data available that are relevant to load research studies and this should facilitate practical use of the approach proposed by utilities when designing load management strategies for peak clipping. Randomizing key model variables together with the use of statistically validated typical water consumption patterns, provides a means of modelling reality with two important results: the contribution of the modelled loads to the load diagram of the region or geographic area under study, and the possibility of anticipating the results of eventual load shedding actions aiming at peak clipping. A key variable is, for example, the water temperature inside the tanks of the modelled EWHs at the beginning of the simulated period of time.

It should be noted that the case study presented, having a small number of consumers and, accordingly, a small number of EWHs, is not representative of the full potential of the methodology described in the paper. Aggregated load models usually require a larger number of controlled loads to become less sensitive to perturbations at the demand side, usually not accounted for in any similar modelling method. As a matter of fact, in spite of accounting for the natural randomness of the use that people make of any energy service, there are implicit conditions - such as the use of dwellings without unexpected interruptions - that, if not actually verified, will cause a certain degree of divergence between model results and reality. Having in mind this type of limitations, usually of lower relative importance for big numbers of consumers, it is evident in any case the possibility of evaluating — both at individual consumer level and at system level — the impacts of LM on the quality of service provided, besides anticipating the impact on the system's load curve.

On the other hand, the application of the proposed method and the underlying simulation tools to a restricted area also mitigates the need of having to define homogeneous groups of loads, common to many other aggregation methods. The authors are presently working towards the validation of the probabilistic assumptions underlying the hot water consumption pattern variations among dwellings within a certain area, through laboratory experiments and data collection in the field.

The method proposed has the advantage of using physically based individual load models (PBILM) that have been validated through experimentation. It allows an effective design of load management (LM) programs through variables such as the number of different load shedding schedules - on their turn informed by time of occurrence and activity duration - and their frequency of use by the utility.

The authors are presently engaged in the use of PBILM in the context of a multiobjective approach to the design of load remote control programs, where maximizing the quality of service to consumers is one of the objectives, among others - only possible because PBILM allows the measuring of this factor. It represents a significant improvement to the iterative 'trial-and-error' procedure of finding the preferred schedule of LM actions, which has been presented in the paper.

\section{ACKNOWLEDGEMENT}

This work was partially funded by the 'Junta Nacional de Investigação Científica e Tecnológica' and by the Portuguese 'Ministério da Ciência e Tecnológica.

\section{REFERENCES}

Alvarez, C., Malhamé, R. P. and Gabaldón, A. (1992). 'A class of models for load management application and evaluation revisited', IEEE Trans. Power Systems, 7, 811-818.

Capasso, A., Grattieri, W., Lamedica, R. and Prudenzi, A. (1994). 'A bottom-up approach to residential load modeling', IEEE Trans. Power Systems, 9, 957-964.

Chong, C. Y. and Debs, A. S. (1979). 'Statistical synthesis of power system functional load models', Proc. IEEE Conf. on Decision and Control, Fort Lauderdale, Florida.

Chong, C. and Malhamé, R. P. (1984). 'Statistical synthesis of physically based load models with applications to cold load pick-up', IEEE Trans. Power Apparatus Systems, PAS-103, 1621-1628.

Dolan, P. S., Nehir, M. H. and Gerez, V. (1996). 'Development of a Monte Carlo based aggregate model for residential electric water heater loads', Electr. Power Systems Res. 36, 29-35. 
Gellings, C. W. and Taylor, R. (1981). 'Electric load curve synthesis - a computer simulation of an electric utility load shape', IEEE Trans. Power Apparatus Systems, PAS-100, 60-65.

Gomes, A. and Gomes Martins, A. (1995). 'Electric load modeling and simulation for assessing DSM actions', Proc. III Int. Congr. of Energy, Environment and Technological Innovation, Caracas, Nov. 1995, 449-454.

Laurent, J., Desauliners, G., Malhamé, R. P. and Soumis, F. (1995). 'A column generation method for optimal load management via control of electric water heaters', IEEE Trans. Power Systems, 10, 1389-1399.

Laurent, J., Malhamé, R. P. (1994). ‘A physically-based computer model of aggregate electric water heating loads', IEEE Trans. Power Systems, 9, 1209-1217.

Lee, S. H. and Wilkins, C. L. (1983). 'A practical approach to appliance load control analysis: a water heater case study', IEEE Trans. Power Apparatus Systems, PAS-102, 1007-1013.

Rau, N. S. and Graham, R. W. (1979). 'Analysis and simulation of the effects of controlled water heaters in a winter peaking system', IEEE Trans. Power Apparatus Systems, PAS-98, 458-464. 